\title{
Beam Steering with a Nonlinear Optical Phased Array Antenna
}

\section{Journal Article}

Author(s):

Busschaert, Sebastian; Flöry, Nikolaus; Papadopoulos, Sotirios; Parzefall, Markus (D); Heeg, Sebastian; Novotny, Lukas (DD

Publication date:

2019-09-11

Permanent link:

https://doi.org/10.3929/ethz-b-000365038

Rights / license:

In Copyright - Non-Commercial Use Permitted

Originally published in:

Nano Letters 19(9), https://doi.org/10.1021/acs.nanolett.9b02029

Funding acknowledgement:

165841 - Nonlinear optics with hybrid plasmonic-TMDC materials (SNF) 


\title{
Beam steering with a nonlinear optical phased array antenna
}

\author{
Sebastian Busschaert, ${ }^{1}$ Nikolaus Flöry, ${ }^{1}$ Sotirios Papadopoulos, ${ }^{1}$ \\ Markus Parzefall, ${ }^{1}$ Sebastian Heeg, ${ }^{1}$ and Lukas Novotny ${ }^{1}$ \\ ${ }^{1}$ Photonics Laboratory, ETH Zürich, Zürich, Switzerland
}

(Dated: February 18, 2020)

\begin{abstract}
Transition metal dichalcogenides (TMDCs) exhibit high second harmonic (SH) generation in the visible due to their non-centrosymmetric crystal structure in odd-layered form and direct bandgap transition when thinned down to a monolayer. In order to emit the $\mathrm{SH}$ radiation into a desired direction one requires a means to control the phase of the in-plane nonlinear polarization. Here, we couple the SH response of a monolayer $\mathrm{MoS}_{2}$ to an optical phased array antenna and demonstrate controllable steering of the nonlinear emission. By exploiting the intrinsic SH generation by the phased array antenna we achieve uniform emission efficiency into a broad angular range. Our work has relevance for novel optoelectronic applications, such as programmable optical interconnects and on-chip LIDAR.
\end{abstract}

Keywords: Nonlinear optics, second harmonic generation, TMDC, phased array antenna, beam steering, LIDAR, optical interconnects

Nonlinear optical effects play a vital role in integrated photonics as frequency mixing and electro-optic modulation are necessary components for optical multiplexing and optoelectronic logic gates $[1,2]$. However, due to the generally weak nonlinear material responses, accessing these effects requires high incident powers. This has lead to an ongoing quest for materials with high intrinsic nonlinearities that can be incorporated into nano-scale devices. In addition, only non-centrosymmetric materials are capable of producing second order nonlinearities in the electric dipole approximation. Unfortunately, common materials such as silicon or gold are centrosymmetric and non-centrosymmetric materials such as lithium niobate are difficult to process on nanometer length-scales [3-5].

Transition metal dichalcogenides (TMDCs) are a new class of materials that possess a strong nonlinear response and that can be structured with top-down nanofabrication techniques. These materials can be thinned down to monolayer form via mechanical exfoliation, which leads to the formation of a direct bandgap semiconductor [6]. Any odd number of layers of TMDCs forms a non-centrosymmetric crystal and has been shown to exhibit high second-order nonlinearities, with reported values up to $\chi^{(2)} \sim 10^{-7} \mathrm{~m} / \mathrm{V}$ for the monolayer form $[7,8]$. Second harmonic $(\mathrm{SH})$ generation has been studied in detail for TMDCs and an extraordinarily high response was observed when the emission was chosen to be close to the optical bandgap of the respective material [9].

Recently, several reports have studied the interaction between a TMDC monolayer and plasmonic particles and demonstrated an enhancement of the $\mathrm{SH}$ response $[10,11]$. The $\mathrm{SH}$ radiation of TMDCs and other material systems can also be controlled by plasmonic and dielectric metasurfaces that shape the phase profile of the incoming light [12-16]. For on-chip optoelectronic applications it is desirable to steer the emission into desired directions and to have programmable control over the emission direction.

As depicted conceptually in Figure 1, we demonstrate a $\mathrm{MoS}_{2}$-gold phased array antenna and demonstrate beam steering of $\mathrm{SH}$ radiation. We utilize the symmetry properties of the array and exploit the interplay between SH generated by $\mathrm{MoS}_{2}$ and the phased array itself to generate a beam steering efficiency that is uniform over a broad angular range.

For the experimental realization, our nonlinear phased array antennas were produced by the following procedure: First, arrays of equally-spaced gold nanorods were fabricated using electron-beam lithography. These arrays were then characterized by scanning electron



FIG. 1. Illustration of a $\mathrm{MoS}_{2}$-gold phased array antenna. 
microscopy (SEM) and by nonlinear spectroscopy. A monolayer of $\mathrm{MoS}_{2}$ was then deposited on top of an array by using a dry transfer technique [17, 18]. The deposited $\mathrm{MoS}_{2}$ interacts with the local optical near fields of the antenna elements and gives rise to $\mathrm{SH}$ generation. Nonlinear measurements before and after transfer of the $\mathrm{MoS}_{2}$ on the same array allows us to quantify the nonlinear signal contributions.

For our $\mathrm{MoS}_{2}$-array design we aimed towards maximizing the nonlinear response and obtaining the desired radiation pattern. In order to achieve a strong nonlinear response we made use of the plasmonic resonance of the gold nanorods constituting the phased array and the electronic resonance of $\mathrm{MoS}_{2}$. The particular dimensions of the rods were chosen such that the plasmonic resonance coincides with the target excitation wavelength at $1310 \mathrm{~nm}$. This resulted in a SH wavelength of $655 \mathrm{~nm}$, which, according to previous studies of $\mathrm{MoS}_{2}$, leads to a strong signal enhancement due to an electronic resonance associated with the A exciton [9].

In Figure 2a we show a scanning electron microscope (SEM) image of a fabricated gold nanorod array on glass and a magnified view. We employed three-dimensional finite element method simulations to calculate the scattering efficiency of the rods, as shown in Figures 2b, c. In Figure $2 \mathrm{~b}$ we depict a plane-cut of the intensity

a

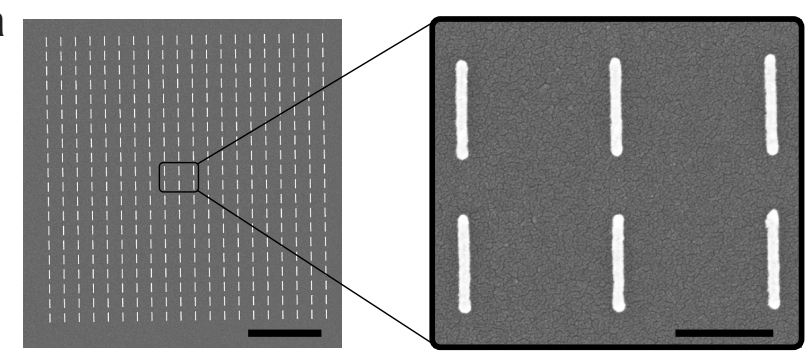

b


FIG. 2. (a) SEM image of a fabricated gold nanorod array on glass (scale bar: $2 \mu \mathrm{m}$ ) including zoomed-in image of the same array (scale bar: $250 \mathrm{~nm}$ ). The designed length was $240 \mathrm{~nm}$. (b) Simulated intensity enhancement of a single gold nanorod $\left(220 \times 30 \times 20 \mathrm{~nm}^{3}\right)$ for $1310 \mathrm{~nm}$ excitation, height of cut is $10 \mathrm{~nm}$, scale bar: $100 \mathrm{~nm}$. (c) Simulated length dependence of the localized surface plasmon polariton (LSPP) resonance of $200 \mathrm{~nm}$ (orange), $220 \mathrm{~nm}$ (red), $240 \mathrm{~nm}$ (black) long nanorods. The lines indicate Lorentzian fits and the green dashed line indicates the target wavelength. The width was fixed to $30 \mathrm{~nm}$ and the height to $20 \mathrm{~nm}$. enhancement of a simulated rod for a $1310 \mathrm{~nm}$ excitation wavelength. The strongest near field regions are at the ends of the nanorod, marking the desired interaction points with the $\mathrm{MoS}_{2}$. By simulating the scattering efficiency for rods with different lengths, shown in Figure 2c, we demonstrate the length dependence of the plasmon resonance in the vicinity of our target wavelength (indicated by the green dashed line). Based on these results we fabricated samples with arrays of varying antenna lengths. In excellent agreement with the simulation we obtain the strongest nonlinear response from nanorod arrays of $220 \mathrm{~nm}$ length (see Supplementary Information), on which we will focus in the following.

The local field of each antenna element (rod) excites a SH response in the overlaid $\mathrm{MoS}_{2}$ and in the rod itself. The SH radiation pattern of the antenna array follows from the coherent superposition of all SH signals. For a phased-array of finite size, the array-characteristic emission pattern is described by the array factor intensity $[19,20]$ :

$$
\begin{aligned}
\operatorname{AF}\left(\phi_{x}, \phi_{y}\right)=\mid \frac{1}{M^{2}} & \frac{\sin 0.5 M\left[k d \sin \phi_{x}+\delta_{x}\right]}{\sin 0.5\left[k d \sin \phi_{x}+\delta_{x}\right]} \\
& \times\left.\frac{\sin 0.5 M\left[k d \sin \phi_{y}+\delta_{y}\right]}{\sin 0.5\left[k d \sin \phi_{y}+\delta_{y}\right]}\right|^{2},
\end{aligned}
$$

where $M$ is the number of antennas in a column/row, $d$ is the distance between antennas, $k=2 \pi n_{\text {glass }} / \lambda_{\mathrm{SH}}$ $\left(n_{\text {glass }}=1.52\right)$ is the wave-vector at the $\mathrm{SH}$ wavelength in glass, $\phi_{x}, \phi_{y}$ are the emission angles and $\delta_{x}, \delta_{y}$ the phase gradients between antennas along $x$ and $y$, respectively. Generally, a larger number of antenna elements (large $M$ ) generates a sharper radiation pattern and the direction of the main radiation lobe can be set by the phase gradients $\delta_{x}$ and $\delta_{y}$. The existence (and number) of radiative grating orders is determined by the spacing $d$. We designed the antenna distance such that no grating orders exist for zero phase delay $\left(\delta_{x}=\delta_{y}=0\right)$ but they can emerge for larger phase delays. This property is a distinct feature of the phased array and will be discussed later on. We investigated phased arrays with different array sizes $M$ and different distances $d$. Here we discuss our results for an array with $20 \times 20$ elements and distance of $d=400 \mathrm{~nm}$.

Nonlinear measurements were performed on the $\mathrm{MoS}_{2}$-array system in a reflection geometry setup (see Supplementary). The array was illuminated with $200 \mathrm{fs}$ pulsed laser light at $1310 \mathrm{~nm}$ through a $1.3 \mathrm{NA}$ oil immersion objective. By focusing the laser onto the center of the back focal plane of the objective we obtain a collimated beam, resulting in an excitation with zero phase delay $\left(\delta_{x}=\delta_{y}=0\right)$. Optical filters were used to separate the $\mathrm{SH}$ emission from the back-reflected excitation. 

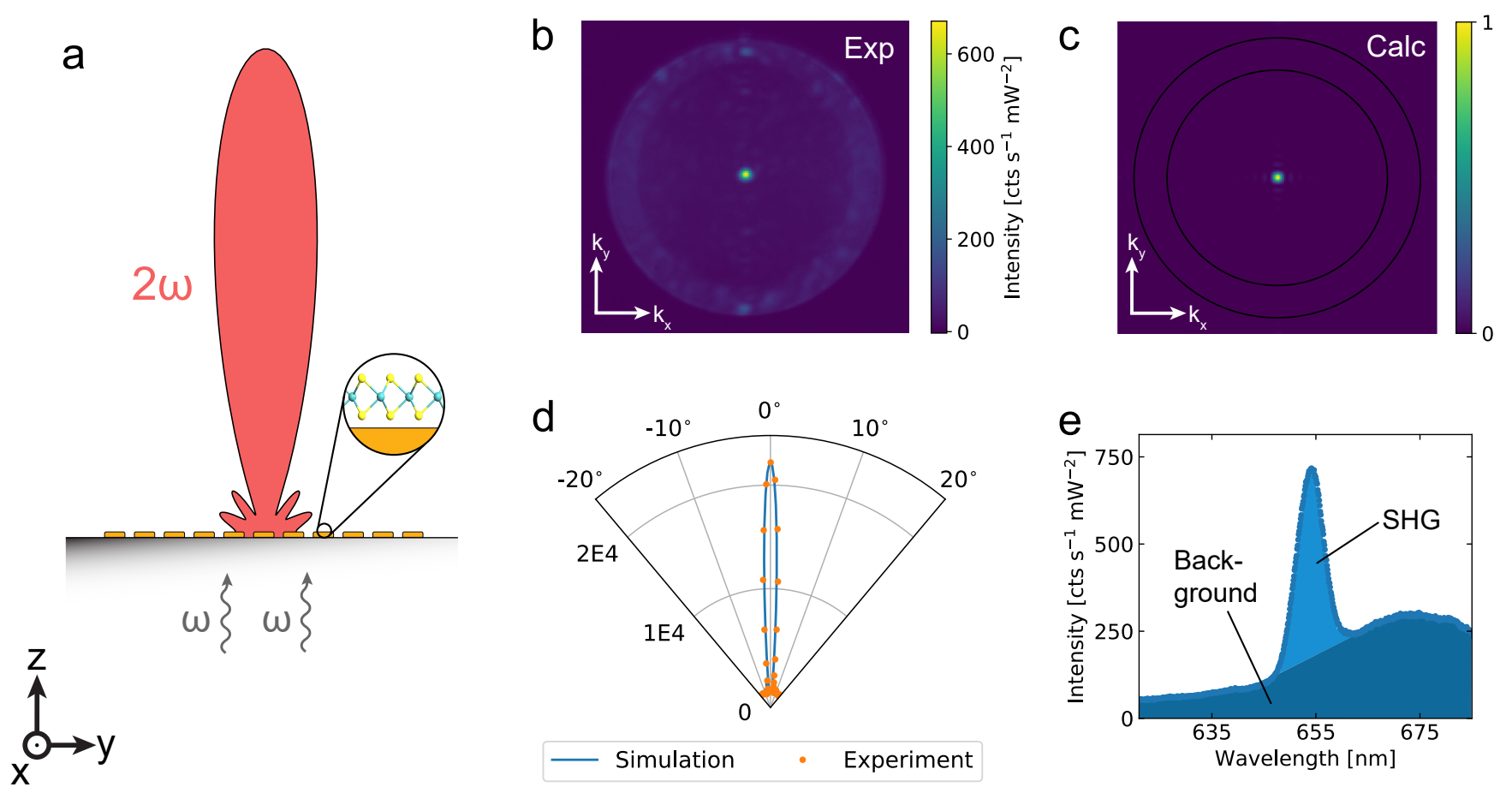

FIG. 3. Nonlinear measurements of the $\mathrm{MoS}_{2}$-array system under normal incidence. (a) Illustration of the studied system. (b) Measured SH emission pattern of the coupled $\mathrm{MoS}_{2}$-array system. The rods are oriented along the y-direction. (c) Calculated SH emission pattern for $2 \mathrm{D}$ array with $M=20$. The black circles represent $k_{\|}=\sqrt{k_{x}^{2}+k_{y}^{2}}=k_{0}$ and $k_{\|}=\mathrm{NA} \times k_{0}$, with $\mathrm{NA}=1.3$ being the numerical aperture of the objective. (d) Polar plot of the calculated (line) and measured (points) SH pattern along the intensity maximum. The intensity is given in units of $\mathrm{cts} \mathrm{s}^{-1} \mathrm{~mW}^{-2} \mathrm{psr}^{-1}$. (e) Spectrum of SH emission (excitation at $1310 \mathrm{~nm}$ ).

The measured $\mathrm{SH}$ radiation pattern of the $\mathrm{MoS}_{2}$-array system is depicted in Figure 3b. We observe a distinct peak at $\left(k_{x}, k_{y}\right)=(0,0)$, corresponding to emission normal to the sample surface, on top of a weaker, nondirectional emission background. The distinct emission spot originates from the constructive interference of the coherent SH emission from individual antenna elements. In Figure 3c we use Eq. (1) to calculate the SH emission pattern as a function of $k_{\|}$, the in-plane wave-vector component, for the case of all array elements radiating in phase $\left(\delta_{x}=\delta_{y}=0\right)$. The measured pattern in Figure 3b is in excellent agreement with the calculation. By taking cross-sections along $k_{x}$ for $k_{y}=0$ and transforming the spatial coordinate into an angular representation we plot in Figure 3d both the calculated and measured emission patterns as a polar plot. There is very good agreement between theory and experiment. We also record the spectrum of the emission from the $\mathrm{MoS}_{2}$-array system in a spectrometer (see Figure 3e), which shows the $\mathrm{SH}$ signal at $655 \mathrm{~nm}$ on top of a spectrally broad background. The background is partially due to the multiphoton luminescence from gold $[21,22]$ (it also appears in measurements on bare gold arrays, see Supplementary) and $\mathrm{MoS}_{2}[23,24]$. Due to its incoherent nature, the background is spectrally broad and non-directional, responsible for the weak background surrounding the sharp SH emission peak.

An important feature of a phased array antenna is the ability to steer the emission and control the change of emission characteristics, such as grating orders. Steering is achieved with the phase gradients $\delta_{x}, \delta_{y}$ in Eq. (1), which define a phase delay between antenna elements. In Figure 4a we illustrate the $\mathrm{SH}$ emission of a two dimensional array for two different phase gradients, which results in emission into angles $\phi=0^{\circ}$ and $\phi=34^{\circ}$, respectively. Experimentally, we achieve a non-zero phase delay by laterally displacing the focus of the excitation beam in the back focal plane of the 1.3 NA oil immersion objective. This leads to a tilted wavefront and hence to a linear phase gradient, as demonstrated in previous studies [25-27].

In our experiments we use $\mathrm{MoS}_{2}$ for nonlinear conversion and the gold phased array to emit the $\mathrm{SH}$ radiation. It turns out, however, that there is a finite $\mathrm{SH}$ contribution from the gold phased array itself [28-30]. In order to distinguish between the two SH contributions we investigate the phased array antenna before and after transfer of $\mathrm{MoS}_{2}$. Our experimental results show that for zero phase delay $\left(\delta_{x}=\delta_{y}=0\right)$ the SH contribution from the gold array is negligible (Figure $4 \mathrm{~b}$ ). The $\mathrm{MoS}_{2}$-array system exhibits the expected emission at $0^{\circ}$ 

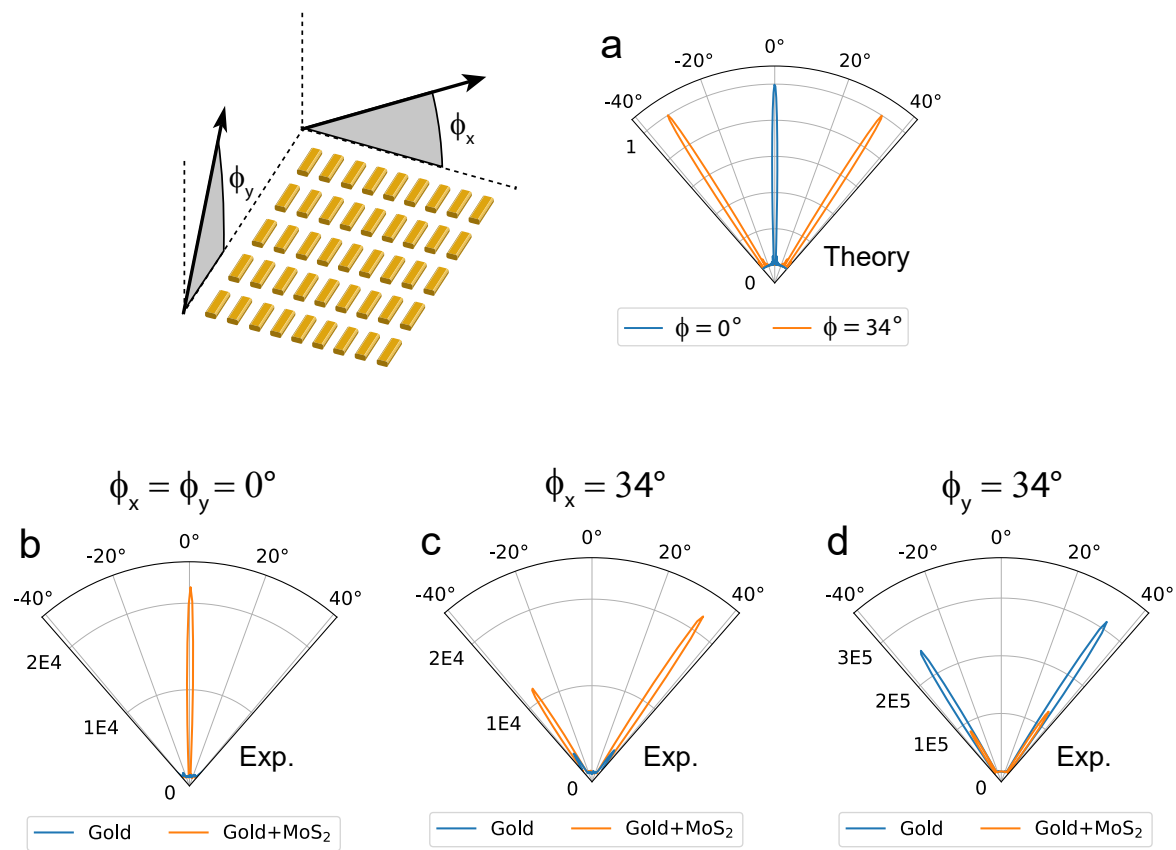

FIG. 4. Beam steering of SH emission by the phase delays along the $x$ direction $\left(\phi_{x}\right)$ and the $y$ direction $\left(\phi_{y}\right)$. The intensity of the measurements is given in units of cts $\mathrm{s}^{-1} \mathrm{~mW}^{-2} \mathrm{\mu sr}^{-1}$. (a) Calculated emission patterns for zero phase delay (blue) and non-zero phase delay (orange) between antenna elements. (b) Measured SH emission of a bare gold array (blue) and a $\mathrm{MoS}_{2}$-array (orange) for zero phase delay. (c,d) Same as (b) but for a phase delay along $x$ yielding $\phi_{x}=34^{\circ}$ (c) and for a phase delay along $y$ yielding $\phi_{y}=34^{\circ}(\mathrm{d})$.

whereas no signal is observed for the bare gold array. The same observation holds for phase delays along the $x$-axis (Figure 4c), which corresponds to a tilt around the axis of a nanorod. However, as shown in Figure 4d, the SH contribution from gold becomes more prominent for large phase delays along the $y$-axis, that is, tilts perpendicular to the nanorod axis. We will analyze this situation later on.

Looking at the emission pattern shown in Figure $4 \mathrm{c}$, we note that there are two emission peaks, one at $34^{\circ}$ (main emission peak) and the other at $-34^{\circ}$ (first grating order). Both of them are predicted by Eq. (1) and indicate the interaction between the gold nanorod array and the $\mathrm{MoS}_{2}$. We attribute the difference in signal strength between the two peaks to the irradiated $\mathrm{MoS}_{2}$ not coupled to the array (in between antennas). In order to quantify the interaction strength we compare $\mathrm{SH}$ generation from the coupled $\mathrm{MoS}_{2}$-array system with SH generation from a bare $\mathrm{MoS}_{2}$ monolayer (see Supplementary). We carry out this comparison for $\delta_{x}=\delta_{y}=0$, as the SH contribution from the gold array can be neglected in this case, cf. Figure 4b. Comparing the absolute intensities of the $\mathrm{MoS}_{2}$-array system and the bare $\mathrm{MoS}_{2}$ only, we determine an enhancement of $E=I_{\mathrm{MoS}_{2} \text {-array }} / I_{\mathrm{MoS}_{2}} \approx 1.6$. This value denotes the area enhancement, that is, the enhancement averaged over many antenna elements. Note that the local signal enhancement at the poles of the nanorods is much larger (see Figure 2b).

In Figure 4d we apply a phase delay along the $y$ direction which coincides with the nanorod axes. In contrast to the previous case, we now observe a significant $\mathrm{SH}$ response from the bare gold array. We thus find that the SH signal emitted in the $(y, z)$ plane is dominated by the gold nanorods whereas the SH signal in the $(x, z)$ plane originates from $\mathrm{MoS}_{2}$. This is a favorable finding as it allows us to emit $\mathrm{SH}$ radiation in different directions with similar efficiency.

Let us analyze why the gold array generates significant $\mathrm{SH}$ radiation only along one tilt axis. Although gold exhibits no bulk SH generation due to its crystal structure, symmetry breaking at surfaces and edges can lead to a non-zero SH contribution [31-34]. The SH emission pattern of a single nanorod is generated by the electric near fields at the ends of the rod (cf. Figure $2 c$ ). The resulting dipoles formed at opposite ends of the rod possess a phase difference of $\pi$, such that their radiation destructively interferes in the farfield at an emission angle of $0^{\circ}$. The two out-of-phase dipoles give rise to a double-lobed SH emission pattern (Figure 5a). Thus, when multiple nanorods are lined up in an array and radiate in phase they destructively interfere at an emission angle of $0^{\circ}$ (Figure $5 \mathrm{~b}$ ). If the incoming beam is tilted such that the main emission lobe is no longer normal to the surface, the destructive interference is lifted 
a

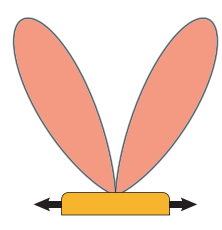

Single rod



$\underset{x \rightarrow y}{z}$

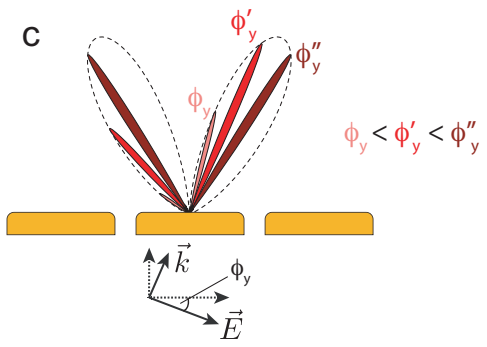

Array with tilted incidence

FIG. 5. Symmetry considerations for $\mathrm{SH}$ generation from a bare gold nanorod array. (a) Long axis view of single nanorod SH emission (only upper half space shown), indicated as red lobes. The emission is rotationally symmetric with respect to the y-axis. The black arrows denote electric dipoles. (b) Long axis view of a nanorod array under normal incidence. Destructive interference cancels the SH emission. (c) Long axis view of a nanorod array under tilted incidence (tilt angle $\left.\phi_{y}\right)$.

(Figure 5c). For this case SH generation is no longer dipole forbidden for observation angles in the $(\mathrm{y}, \mathrm{z})$ plane.

In order to confirm the explanation described in Figure 5 we changed the phase delay along the two symmetry axes in small increments and tracked the SH intensity of the main emission lobe. In Figure 6a we show the behavior of the bare gold array, first for the $y$-direction $\left(\phi_{y}\right)$ and then the $x$-direction $\left(\phi_{x}\right)$. For zero phase delay $\left(\delta_{x}=\delta_{y}=0\right)$ we do not observe any significant $\mathrm{SH}$ contribution, however as we increase the phase delay along $y$ the $\mathrm{SH}$ emission appears and increases with increasing phase delay. For the $x$ direction, on the other hand, there seems to be only a small, negligible $\mathrm{SH}$ contribution (note the different scales in the plots). The integrated values for several emission angles are compiled in Figure $6 \mathrm{~b}$ and normalized to the highest measured $\mathrm{SH}$ value. The values along $x$ are up to nearly 3 orders of magnitude smaller than along $y$ and remain constant until the maximum applied phase delay. In order to understand the increase of SH generation along $y$ we consider two dipoles at opposite ends (as illustrated in Figure 5a) and calculate the radiation pattern of a single nanorod as a function of solid angle $I_{\text {quad,em }}(\phi)$. We account for the fact that the local excitation intensity is angle-dependent (due to the array) $I_{\text {exc }}(\phi)$. The same is true for the transmission of the excitation through the sample $I_{\text {trans }}(\phi)$ (for more details see Supplementary).
We then obtain the angle-dependent $\mathrm{SH}$ intensity of a single nanorod as

$$
I_{\text {antenna }}(\phi)=I_{\text {quad,em }}(\phi) I_{\text {exc }}(\phi) I_{\text {trans }}(\phi)
$$

The result of this calculation is shown as a dashed line in Figure $6 \mathrm{~b}$ and agrees well with the data points.

In order to obtain the complete $\mathrm{SH}$ emission pattern of the full array, the expression has to be multiplied with the array factor in Eq. (1)

$$
I_{\text {phased array }}(\phi)=\operatorname{AF}(\phi) I_{\text {antenna }}(\phi)
$$

For the $\mathrm{MoS}_{2}$-array system we are not only dealing with the nonlinearity of the bare gold array (which we denote by $I_{\text {quad,em }}(\phi)$ ) but also by the nonlinearity of the $\mathrm{MoS}_{2}$. The same analysis as for the bare gold array was conducted for the $\mathrm{MoS}_{2}$-array system and the results are shown in Figures $6 \mathrm{c}$ and d. Compared to the bare gold array, the overall signal strength is weakened for a phase delay along the $y$-direction $\left(\phi_{y}\right)$ but the qualitative behavior is similar. For phase delays along the $x$ direction we observe that the $\mathrm{SH}$ signal is constant for a wide range of angles $\phi_{x}$ and only shows a small increase for a large $\phi_{x}$. This observation agrees with the explanation given in Figure 5, which predicts that the emission along the $x$ direction is solely due to $\mathrm{SH}$ generation by $\mathrm{MoS}_{2}$.

In Figure 6d we show the integrated $\mathrm{SH}$ signal as a function of emission angle. We observe that the signal strengths along the two symmetry axes differ only by one order of magnitude (for the bare gold array the difference was nearly three orders of magnitude, c.f. Figure 6b). This favorable behavior arises from the fact that both $\mathrm{MoS}_{2}$ and the gold array contribute to the nonlinear signal generation. In order to describe our measured data we use Eq. (2) with the addition of a dipolar emission pattern that describes the response of the $\mathrm{MoS}_{2}$ (see Supplementary):

$$
\begin{aligned}
I_{\text {antenna }}(\phi)=\left|E_{\text {quad }, \mathrm{em}}(\phi)+E_{\text {dipole,em }}(\phi)\right|^{2} \\
\times I_{\text {exc }}(\phi) I_{\text {trans }}(\phi)
\end{aligned}
$$

The resulting theoretical curve agrees well with the data points and explains the two different $\mathrm{SH}$ contributions $\left(\mathrm{MoS}_{2}\right.$ and gold array) in the measured emission patterns.

We finally use the $\mathrm{MoS}_{2}$-array system for dynamic beam steering and compare it with the bare gold array. We used a motorized lens-system that tilts the incoming wavefront in the sample plane, thereby dynamically changing the applied phase delay. Using this approach we projected the pattern "ETH" into the farfield. This 



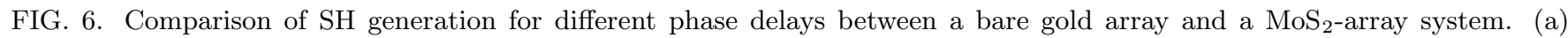
Measured SH emission patterns of a bare gold array for three different phase delays along the $y$-direction (left) and the $x$ direction (right). The intensity is given in units of $\operatorname{cts~s}^{-1} \mathrm{~mW}^{-2} \mathrm{psr}^{-1}$. (b) Integrated SH intensities as a function of emission angle. The data is normalized to the value measured at $\phi_{y}=34^{\circ}$ and absolute values are given for comparison with the coupled system. The dashed orange line corresponds to a theoretical model, as explained in more detail in the main text. (c,d) Corresponding results for the combined $\mathrm{MoS}_{2}$-array system. The dashed blue line corresponds to a theoretical model described by the transmission function of the excitation. The emission efficiency is more uniform for the case of the MoS2-array system.

pattern, shown in Figure 7, was imaged with a CCD camera with an integration time of 50 seconds. As demonstrated in Figure 7a, writing with the bare gold array results in the horizontal writing line missing due to the symmetry forbidden region. However, when we write the same sequence with the $\mathrm{MoS}_{2}$-array system, shown in Figure 7b, we profit from $\mathrm{SH}$ generation from $\mathrm{MoS}_{2}$. The resulting farfield pattern now shows the full "ETH" logo, including all vertical and horizontal lines.
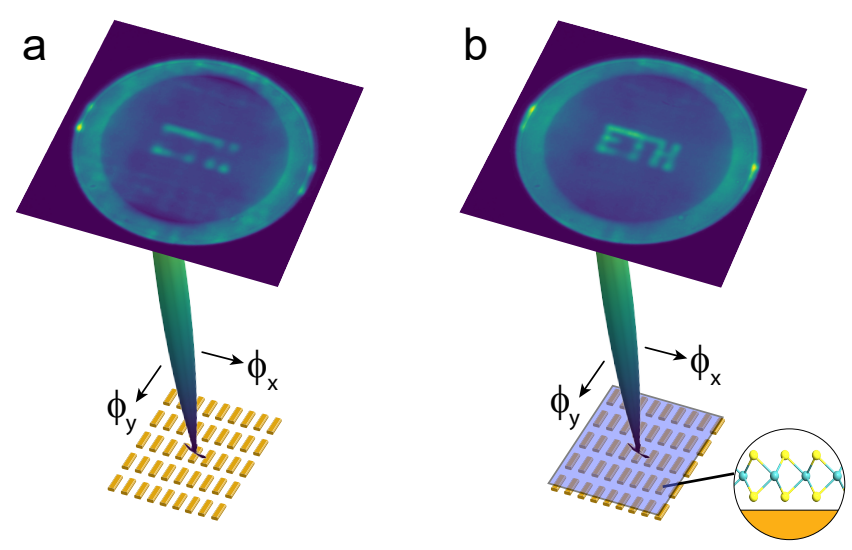

FIG. 7. Dynamic beam steering of SH emission. The phase delays along $\phi_{x}$ and $\phi_{y}$ were varied in time to write a "ETH" farfield pattern. (a) Resulting pattern for the bare gold array. (b) Resulting pattern for the $\mathrm{MoS}_{2}$-array system.
Thus, the combined $\mathrm{MoS}_{2}$-array system allows us to steer $\mathrm{SH}$ radiation into arbitrary directions.

In conclusion, we showed the nonlinear coupling of a monolayer semiconductor, here $\mathrm{MoS}_{2}$, to an array of gold nanorods. The combined system functions as a phased array antenna that emits $\mathrm{SH}$ radiation into arbitrary directions. Beam steering is accomplished by controlling the phase delay between antenna elements along the two main axes. The emission efficiency is not only dictated by the array factor, but also by the emission pattern of a single antenna element. The presented results offer a new platform to study and understand the coupling of second order nonlinear effects mediated by TMDCs and structured surfaces. Furthermore, nonlinear optical phased array antennas can have applications for dynamical optical interconnects and on-chip LIDAR.

\section{METHODS}

\section{Electrodynamic Simulations}

Numerical simulations were carried out with the RF module of COMSOL Multiphysics. A single gold rod with dimensions of $30 \mathrm{~nm}$ width, $20 \mathrm{~nm}$ height and variable length was defined on top of a glass substrate ( $\mathrm{n}$ $=1.52)$, whereas the rod was surrounded by air $(\mathrm{n}=$ 1) in the other half space. For the dielectric function 
of gold we used measured values [35]. The simulations were carried out in two steps: First, the fields of the geometry were simulated under plane wave illumination without rod such that the background field could be determined. The two half spaces were surrounded by perfectly matched layers (PMLs) to exclude back-reflection. In a a second step, the geometry was simulated with rod. The scattering efficiency of a single rod was calculated by normalization with the cross-section of the rod. For the final scattering efficiency comparison between different rods, we normalized the scattering efficiencies to the highest simulated value for the $240 \mathrm{~nm}$ rod. Comparing the resonance condition of a single rod with the measured resonance of the full array did not show any significant deviations, which is due to the large separation between antenna elements (negligible near field interaction between antenna elements).

\section{Sample Fabrication}

Phased array antennas were fabricated on commercial glass coverslips. First, markers were patterned by means of electron beam lithography (EBL), evaporation of $5 \mathrm{~nm}$ titanium (Ti, $0.05 \mathrm{~nm} / \mathrm{s}$ ) and $50 \mathrm{~nm}$ gold ( $\mathrm{Au}$, $0.2 \mathrm{~nm} / \mathrm{s}$ ) at a pressure of $<10^{-7} \mathrm{mbar}$ and subsequent lift-off (acetone, ispropyl alcohol and deionized water). The antenna arrays consisting of $\mathrm{Au}$ nanorods were then defined again by EBL as single pixel lines (SPL, line-dose $1400 \mathrm{pAs} / \mathrm{cm}$ ), followed by development and soft plasma cleaning $\left(\mathrm{O}_{2}\right.$ plasma, $\left.70 \mathrm{~W}, 30 \mathrm{~s}\right) .20 \mathrm{~nm} \mathrm{Au}$ was deposited by electron beam evaporation $(0.1 \mathrm{~nm} / \mathrm{s})$ at a pressure of $<10^{-7} \mathrm{mbar}$, followed by lift-off. For scanning electron microscopy a sample fabricated with the same parameters was sputter-coated with $1.5 \mathrm{~nm}$ $\mathrm{Pt} / \mathrm{Pd}$ and then imaged using the in-lens detector (TLD) of a FEI Magellan 400 system.

For the final structure including the monolayer $\mathrm{MoS}_{2}$, bulk $\mathrm{MoS}_{2}$ crystal was exfoliated onto UV-ozone cleaned polydimethylsiloxane (PDMS). After observing a monolayer via optical contrast difference, the respective flake was stamped on top of a suitable array with $\mu \mathrm{m}$ precision using a SUSS MicroTec mask aligner.

\section{Nonlinear Measurements}

For the nonlinear measurements we used the IR output of an optical parametric oscillator (Coherent Mira-OPO) that provided $200 \mathrm{fs}$ pulses at a repetition rate of $76 \mathrm{MHz}$. All experiments were conducted with $1310 \mathrm{~nm}$ light. To exclude any pump contributions we spectrally filtered the laser (Semrock BL 1110LP, Chroma HHQ940LP). The beam was sent into a $90^{\circ}-$ periscope consisting of two motorized stages (PT1/M-Z8 by Thorlabs) and an attached lens (AC254-400-C-ML by Thorlabs), that enabled us to focus the laser onto the back focal plane (BFP) of an oil immersion objective (1.3 NA Plan Fluor Nikon) and to laterally displace the beam. Focusing the laser onto the BFP leads to a large area illumination of the sample and lateral displacement of the beam gives rise to a wavefront tilt and hence a phase delay between antenna elements. With an initial beam waist of $2 \mathrm{~mm}$, the beam spot diameter in the sample plane was roughly $10 \mu \mathrm{m}$. The average power before entering the periscope was determined to be $1.6 \mathrm{~mW}$, resulting in an average power of 0.83 $\mathrm{mW}$ on the sample. The excitation peak intensity never exceeded $1 \mathrm{GW} / \mathrm{cm}^{2}$, which is below the damage threshold for the gold nanorods. The incident polarization was chosen to be along the long axis of the nanorods.

The excitation and emission wavelengths were seperated by a dichroic beamsplitter (DMSP950R by Thorlabs), further spectral selection was performed via optical filters (770SP, 650/60BP for SH generation, $561 \mathrm{SP}$ for THG, all filters by Semrock) in front of the detectors. The emission was sent either to a spectrometer (Acton SP2300 with Pixis100 CCD) or an EMCCD (Acton Photon Max 512). Different lens arrangements were used for real space and Fourier space imaging.

For calibration of the emission angles we used the first concentric ring in the $\mathrm{BFP}\left(\mathrm{NA}=1\right.$ or $\left.\phi=41.14^{\circ}\right)$ for normalization. The error in determining the angle is $\pm 1^{\circ}$ (see Supplementary Information for more details).

\section{ACKNOWLEDGMENTS}

This work was financially supported by the Swiss National Science Foundation (grant no. 200021_165841). S. Heeg acknowledges financial support by ETH Zurich Career Seed Grant SEED-16 17-1. The authors thank A. Jain for valuable input during sample fabrication and D. Windey for providing support during data analysis. Furthermore, the authors acknowledge the use of the facilities of FIRST center of micro- and nanoscience and ScopeM at ETH Zürich.

\section{SUPPORTING INFORMATION}

The Supporting Information includes:

Nonlinear setup schematic; additional characterization of bare gold arrays and $\mathrm{MoS}_{2}$-array system; explanation of polar plot representation; equations for the individual antenna emission patterns; measurement comparison for plasmonic enhancement calculation; measurements with non-resonant excitation; noise study of k-space measurements. 


\section{REFERENCES}

[1] L.-W. Luo, N. Ophir, C. P. Chen, L. H. Gabrielli, C. B. Poitras, K. Bergmen, and M. Lipson, Nature Communications 5, 3069 EP (2014).

[2] C. Reis, T. Chattopadhyay, P. André, and A. Teixeira, Appl. Opt. 51, 8693 (2012).

[3] R. W. Boyd, Nonlinear Optics (Elsevier, 2008).

[4] R. L. Sutherland, Handbook of Nonlinear Optics, 2nd ed., Optical engineering, Vol. 82 (Marcel Dekker, New York, 2003).

[5] S. Y. Siew, E. J. H. Cheung, H. Liang, A. Bettiol, N. Toyoda, B. Alshehri, E. Dogheche, and A. J. Danner, Opt. Express 26, 4421 (2018).

[6] K. F. Mak, C. Lee, J. Hone, J. Shan, and T. F. Heinz, Phys. Rev. Lett. 105, 136805 (2010).

[7] N. Kumar, S. Najmaei, Q. Cui, F. Ceballos, P. M. Ajayan, J. Lou, and H. Zhao, Phys. Rev. B 87, 161403 (2013).

[8] L. M. Malard, T. V. Alencar, A. P. M. Barboza, K. F. Mak, and A. M. de Paula, Phys. Rev. B 87, 201401 (2013).

[9] M. Zhao, Z. Ye, R. Suzuki, Y. Ye, H. Zhu, J. Xiao, Y. Wang, Y. Iwasa, and X. Zhang, Light: Science \&Amp; Applications 5, e16131 (2016).

[10] Z. Wang, Z. Dong, H. Zhu, L. Jin, M.-H. Chiu, L.-J. Li, Q.-H. Xu, G. Eda, S. A. Maier, A. T. S. Wee, C.-W. Qiu, and J. K. W. Yang, ACS Nano 12, 1859 (2018).

[11] J. Shi, W.-Y. Liang, S. S. Raja, Y. Sang, X.-Q. Zhang, C.-A. Chen, Y. Wang, X. Yang, Y.-H. Lee, H. Ahn, and S. Gwo, Laser \& Photonics Reviews 12, 1800188 (2018).

[12] J. Chen, K. Wang, H. Long, X. Han, H. Hu, W. Liu, B. Wang, and P. Lu, Nano Letters 18, 1344 (2018).

[13] G. Hu, X. Hong, K. Wang, J. Wu, H.-X. Xu, W. Zhao, W. Liu, S. Zhang, F. Garcia-Vidal, B. Wang, P. Lu, and C.-W. Qiu, Nature Photonics (2019).

[14] F. J. F. Löchner, A. N. Fedotova, S. Liu, G. A. Keeler, G. M. Peake, S. Saravi, M. R. Shcherbakov, S. Burger, A. A. Fedyanin, I. Brener, T. Pertsch, F. Setzpfand, and I. Staude, ACS Photonics 5, 1786-1793 (2018).

[15] G. Marino, C. Gigli, D. Rocco, A. Lemaître, I. Favero, C. De Angelis, and G. Leo, ACS Photonics 6, 1226-1231 (2019).

[16] P. P. Vabishchevich, A. Vaskin, S. Addamane, S. Liu, A. P. Sharma, G. Balakrishnan, J. L. Reno, G. A. Keeler,
M. B. Sinclair, I. Staude, and I. Brener, Conference on Lasers and Electro-Optics, FM2C.3 (2019).

[17] A. Castellanos-Gomez, M. Buscema, R. Molenaar, V. Singh, L. Janssen, H. S. Van Der Zant, and G. A. Steele, 2D Materials 1, 011002 (2014).

[18] A. Jain, P. Bharadwaj, S. Heeg, M. Parzefall, T. Taniguchi, K. Watanabe, and L. Novotny, Nanotechnology 29, 265203 (2018).

[19] T. A. Milligan, Modern Antenna Design (Wiley, 2005).

[20] C. A. Balanis, Antenna theory: analysis and design (Wiley-Interscience, 2005).

[21] T. Wang, D. Halaney, D. Ho, M. D. Feldman, and T. E. Milner, Biomed. Opt. Express 4, 584 (2013).

[22] S. Wang, W. Xi, F. Cai, X. Zhao, Z. Xu, J. Qian, and S. He, Theranostics 5, 251 (2015).

[23] Y. Li, N. Dong, S. Zhang, X. Zhang, Y. Feng, K. Wang, L. Zhang, and J. Wang, Laser \& Photonics Reviews 9, 427 (2015).

[24] F. Zhou and W. Ji, Opt. Lett. 42, 3113 (2017).

[25] G. Ghielmetti and C. M. Aegerter, Opt. Express 20, 3744-3752 (2012).

[26] D. Dregely, K. Lindfors, M. Lippitz, N. Engheta, M. Totzeck, and H. Giessen, Nature Communications 5, 4354 SP (2014).

[27] E. Almeida, G. Shalem, and Y. Prior, Nature Communications 7, $10367 \mathrm{SP}$ (2016).

[28] M. D. McMahon, R. Lopez, R. F. Haglund, E. A. Ray, and P. H. Bunton, Phys. Rev. B 73, 041401 (2006).

[29] D. C. Hooper, C. Kuppe, D. Wang, W. Wang, J. Guan, T. W. Odom, and V. K. Valev, Nano Letters 19, 165 (2019).

[30] S. M. S. Hamed, L. Robert, W. Feng, D. A. Eshaghian, T. Hossein, E. A. A., H. Hayk, and A. Ali, Nanophotonics 8, 607 (2019).

[31] A. Bouhelier, M. Beversluis, A. Hartschuh, and L. Novotny, Phys. Rev. Lett. 90, 013903 (2003).

[32] G. Bachelier, J. Butet, I. Russier-Antoine, C. Jonin, E. Benichou, and P.-F. Brevet, Phys. Rev. B 82, 235403 (2010).

[33] P. Reichenbach, L. M. Eng, U. Georgi, and B. Voit, Journal of Laser Applications 24, 042005 (2012).

[34] M. Kauranen and A. V. Zayats, Nature Photonics 6, 737 EP (2012).

[35] P. B. Johnson and R. W. Christy, Phys. Rev. B 6, 4370 (1972). 


\title{
Supporting Information: Beam steering with a nonlinear optical phased array antenna
}

\author{
Sebastian Busschaert, ${ }^{1}$ Nikolaus Flöry, ${ }^{1}$ Sotirios Papadopoulos, ${ }^{1}$ \\ Markus Parzefall, ${ }^{1}$ Sebastian Heeg, ${ }^{1}$ and Lukas Novotny ${ }^{1, *}$ \\ ${ }^{1}$ Photonics Laboratory, ETH Zürich, Zürich, Switzerland
}

\section{NONLINEAR SETUP}



Fig. S 1. Illustration of setup for nonlinear measurements. The beam path starts at the optical parametric oscillator (Coherent Mira-OPO) that produces $200 \mathrm{fs}$ pulses with a repitition rate of $76 \mathrm{MHz}$. The laser is subsequently spectrally filtered with the following components: Semrock BL 1110LP, Chroma HHQ940LP. Afterwards the beam enters a periscope (mirrors mounted at $90^{\circ}$ angle) that is motorized using two translation stages (PT1/M-Z8, Thorlabs) and connected to a lens (AC254-400-C-ML, Thorlabs). The beam gets reflected at a dichroic beamsplitter (DMSP950R by Thorlabs) and enters an oil immersion objective (1.3 NA Plan Fluor 100x Nikon) after which it hits the sample and produces the nonlinear signal. The signal passes the dichroic beamsplitter and can either be send to a spectrometer (Acton SP2300 with Pixis100 CCD) or to an EMCCD (Acton Photon Max 512) by using a flip mirror. The EMCCD is able to image either the real space of the sample or the back focal plane of the objective by choosing an appropriate lens before. Before the detectors, additional filters (770SP, 650/60BP for SHG, 561SP for THG, all filters by Semrock) only pass the wanted nonlinear signal. Abbreviations: LP - longpass filter, SP - shortpass filter, BP - bandpass filter, BS - beamsplitter, BFP - back focal plane.

For the nonlinear measurements the attached lens mounted to the motorized periscope enabled us to focus the laser onto the back focal plane (BFP) of the oil immersion objective and thus created a collimated illumination at the sample stage. Since the resulting beamspot in the sample plane $(\approx 10-15 \mu \mathrm{m})$ is larger than the array size $(\approx 8 \mu \mathrm{m})$, the illumination leads to an excitation of the nanorods with zero phase delay. Any lateral displacement of the focused beam from the optical axis leads to a tilt in the exciting wavefront (illustrated in Figure S1). The tilted wavefront leads to a linear phase gradient in the excitation of the nanorods, thus constituting a non-zero phase delay. It was paramount to both move the beam and the lens simultaneously (by attaching the lens to the periscope) in order to ensure non-distorted tilting of the wavefront. Only moving the lens or tilting the beam before the lens would have

\footnotetext{
*lnovotny@ethz.ch
} 
resulted in an axial displacement and hence an uneven illumination of the whole array.

In order to write ETH a script for the motorized stages was implemented using the Kinesis software from Thorlabs. The script consisted of sequential movements perpendicular to the optical axis, that were captured by the EMCCD using a longer integration time (50 seconds). 


\section{CHARACTERIZATION}
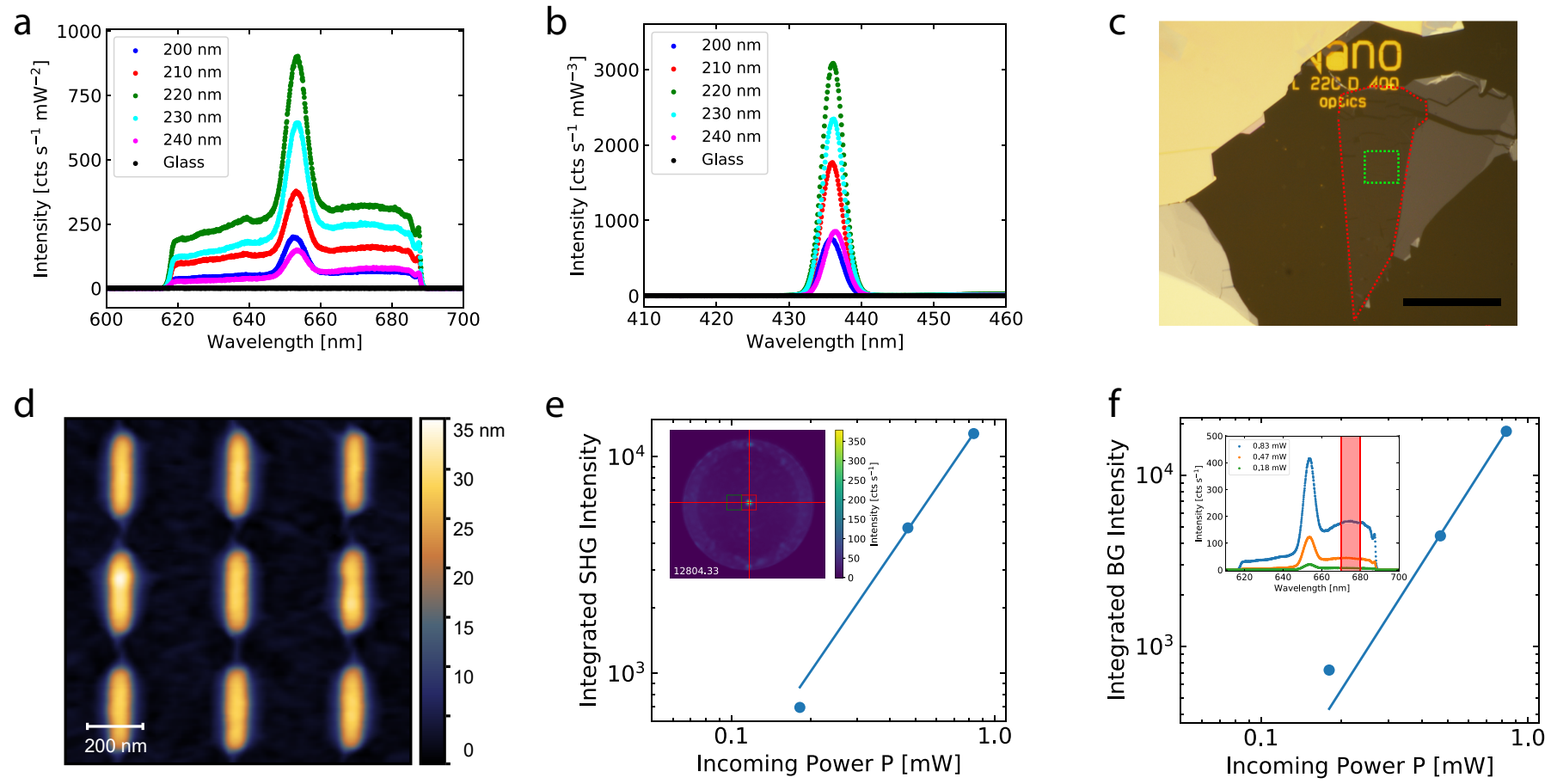

Fig. S 2. Characterization of bare gold arrays and final $\mathrm{MoS}_{2}$-array device. (a,b) SHG, THG responses of arrays for various rod lengths. The $220 \mathrm{~nm}$ rods scatter the strongest. (c) Bright field image of final device. The monolayer $\mathrm{MoS}_{2}$ is indicated with a red dashed line, the array with a green dashed line. The scale-bar is $25 \mu \mathrm{m}$. (d) AFM scan of the coupled system, demonstrating the folding of the $\mathrm{MoS}_{2}$ over the rods. (e) Power-dependent integrated SHG intensities of the coupled MoS ${ }_{2}$-array system. The data was evaluated by summing over a certain pixel area in the recorded BFP images (see inset, the green box indicates the background data that was subtracted). The line indicates a fit corresponding to $I(P)=A \times P^{1.8 \pm 0.1}$, thus agreeing with the expected nonlinear order. (f) Power-dependent integrated background (BG) intensities of the coupled MoS ${ }_{2}$-array system. The data was evaluated by integrating the respective spectra, see inset (red box indicates the integration boundaries). The line corresponds to a fit with a functional form of $I(P)=A \times P^{2.4 \pm 0.1}$, which indicates a mix of multiphoton processes. 


\section{POLAR PLOT REPRESENTATION}
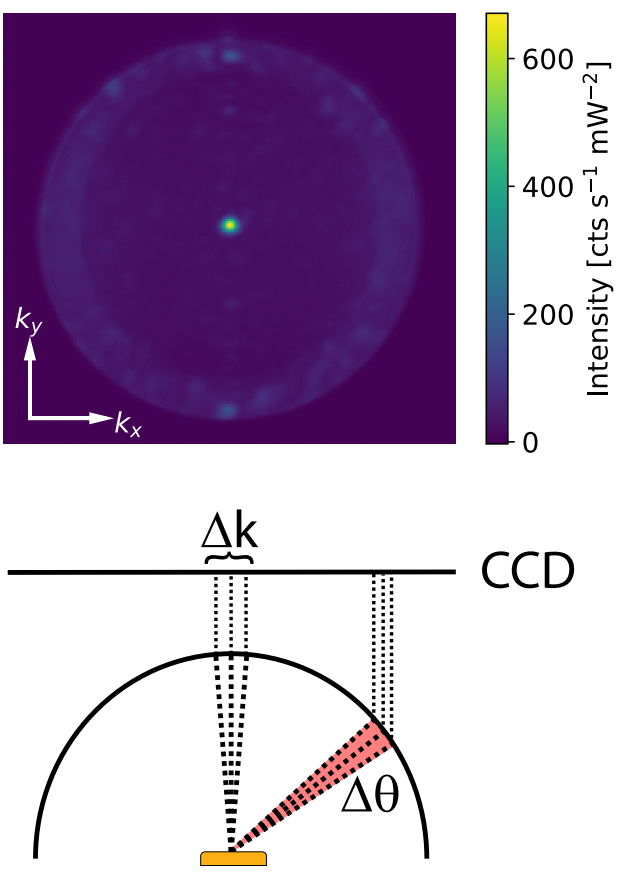

Fig. S 3. Angular projection of the emission on the CCD plane. Depending on the radiation angle with respect to the rod, the same angular spread $\Delta \phi$ can lead to a different $\Delta k$.

Imaging the back focal plane of the 1.3 NA oil immersion objective onto a CCD leads to a projection of the k-space, which can be transformed into an emission angle $\phi$-dependent polar plot representation. The recorded image is linear in pixels, i.e. $k_{x, y}$, and gives us an emission intensity with the unit counts per $\Delta k$ - the transformation into an emission with unit counts per $\Delta \phi$ requires the casting of $\frac{1}{\Delta k}$ into $\frac{1}{\Delta \phi}$. This is not a linear transformation as illustrated in Figure S3. Since we project onto a circle (i.e. collection lens), $\Delta k$ is bigger for small emission angles and decreases for larger emission angles. This leads to higher recorded intensity per pixel since the emission is captured by less pixels. In order to account for that, we used the inner ring in the recorded images (dashed line) as a normalization, since

$$
\phi_{N A=1}=\arcsin \frac{n_{\text {air }}}{n_{\text {glass }}}=41.14^{\circ}
$$

With that we could assign an angle $\phi_{N A=1}$ to certain pixel values, construct an array of $k_{x, y}$ values in between $\left(k_{x, y} \sim p x\right)$ and then calculate the corresponding angle values using

$$
\phi=\arcsin \frac{k_{x, y}}{k}
$$

The wavevector $\mathrm{k}$ is given by $k=\frac{2 \pi}{\lambda_{S H G}}$. By then calculating $\Delta \phi=\phi_{i+1}-\phi_{i}$ we arrive at the desired transformation with unit $\operatorname{deg}^{-1}$. Additionally, the pixels of the detector also have a finite size orthogonal to the evaluated emission direction. By multiplying with the angle-transformed pixel width at the center of the CCD an expression with the unit $\mathrm{deg}^{-2}$ is generated. To comply with SI unit standards we transform $\mathrm{deg}^{-2}$ into $\mathrm{usr}^{-1}$. 


\section{ANTENNA EMISSION PATTERN}

For the angle dependency of the emission we used the analytical far field solution of two opposite dipoles:

$$
I_{\text {quad }, \mathrm{em}}=4\left|\cos \phi \sin \frac{\pi d \sin \phi}{\lambda}\right|^{2}
$$

Here $d$ is the separation between the dipoles and is given by the rod length $d=220 \mathrm{~nm}$. The wavelength was chosen to be $\lambda=\frac{655 \mathrm{~nm}}{1.52}$ since the system sits on glass $\left(n_{\text {glass }}=1.52\right)$ and we observe the emission through an oil immersion objective with the same refractive index.

The excitation is described by a term that describes the angle-dependent plasmon resonance

$$
I_{\text {exc }}=\left(\cos ^{2} \phi\right)^{2}
$$

The term is to the power of 2 due to the nonlinear order of SHG.

The second term for the excitation is due to a change of the intensity when changing the incoming wavefront. This effect is the inverse problem to the angular representation discussed in Figure S3. The focus in the back focal plane of the objective remains constant in size, but with larger displacement from the center (and larger angular spread) the light is sent onto a smaller area in the sample plane. The incoming power remains constant throughout - hence, for larger angles the intensity increases according to:

$$
I_{\text {trans }}=\frac{1}{\cos ^{2} \phi}
$$

For the expression for the $\mathrm{MoS}_{2}$-array system we added a term that describes the dipolar emission characteristic of the TMDC. Since the term for the opposite dipoles and the dipolar term can interfere with each other, we added the respective electric fields and then squared the expression

$$
\left|E_{\text {quad }, \mathrm{em}}+E_{\text {dipole,em }}\right|^{2}=\left|\cos \phi\left(1-2 i \sin \frac{\pi d \sin \phi}{\lambda}\right)\right|^{2}
$$

Both final expressions were then fitted to the data, the bare array with one fitting parameter, the $\mathrm{MoS}_{2}$-array system with two parameters. 


\section{ENHANCEMENT}

a
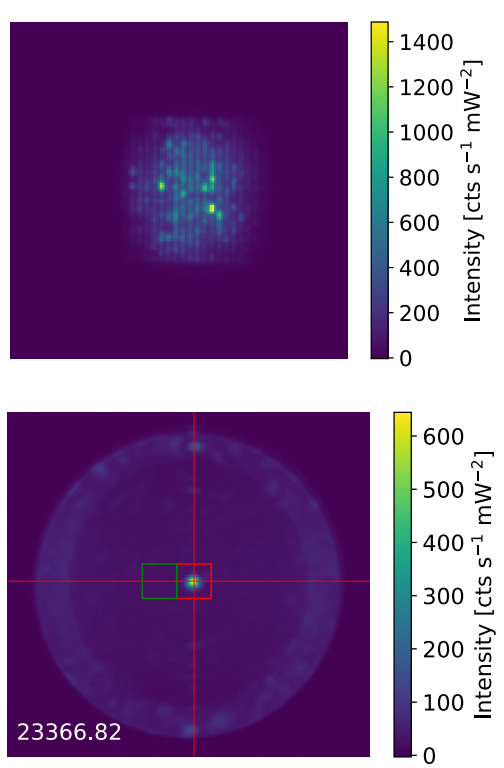

$\mathrm{b}$
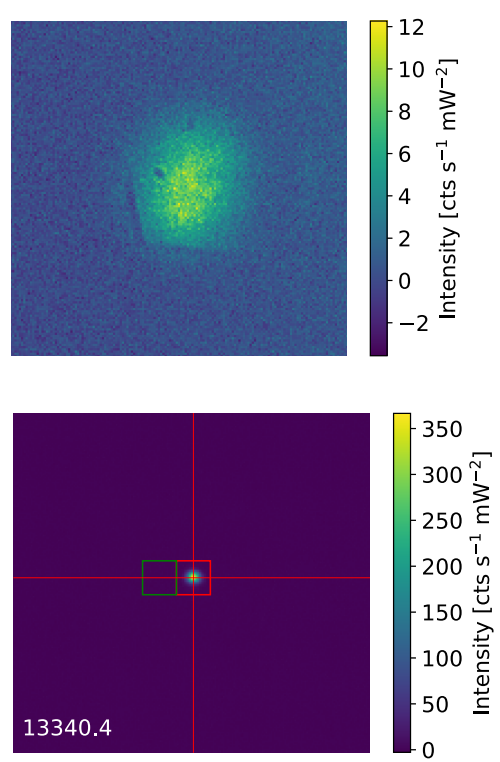

C
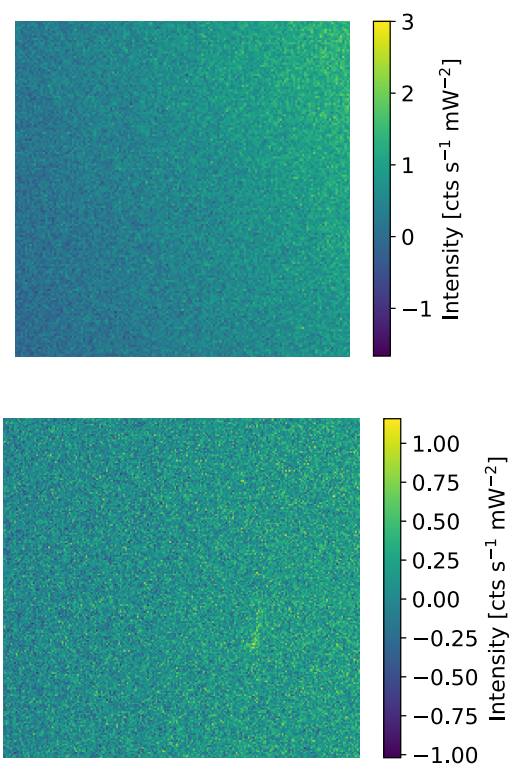

Fig. S 4. SHG enhancement of $\mathrm{MoS}_{2}$-array system. Measured SHG real space and back focal plane (BFP) images of (a) $\mathrm{MoS}_{2}$-array system, (b) bare $\mathrm{MoS}_{2}$ and (c) glass. The emission feature in the center of the BFP images is integrated, the red box indicates the area (20x20 pixels) of the integrated signal $S$ and the green box the integrated area for background subtraction $S_{b g}$. The white number indicates the total nonlinear signal $I=S-S_{b g}$. This measurement was repeated three times with slightly varying beam spot placement, resulting in a calculated SHG enhancement of $\frac{I_{s y s t e m}}{I_{M o S_{2}}} \approx 1.6$. 
VI. NON-RESONANT EXCITATION


Fig. S 5. SHG measurements of $\mathrm{MoS}_{2}$-array system with the incident polarization non-resonant with the rods (sample was rotated by $90^{\circ}$ ) (a) Dark field microscope image of measured system. Two cracks in the MoS $\mathrm{M}_{2}$ above the array are visible, giving a distinct feature for orientation. Scale bar: $10 \mu \mathrm{m}$ (b) Real space and back focal plane (BFP) images of SHG under normal incidence. The cracks observed in (a) are seen in the real space image. (c,d) Real space and BFP images under tilt which is equivalent to a tilt in $\phi_{y}, \phi_{x}$ direction as assigned in the main text. In contrast to the resonant excitation (described in the main text), no grating orders are observed under tilt for non-resonant excitation. 


\section{NOISE STUDY OF K-SPACE MEASUREMENTS}

a

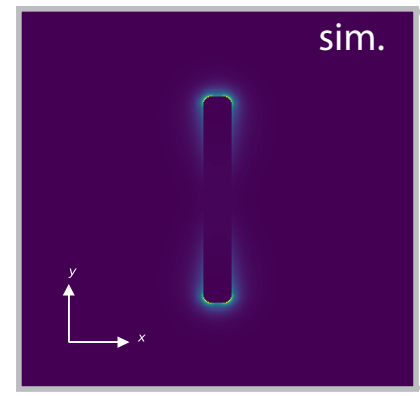

d

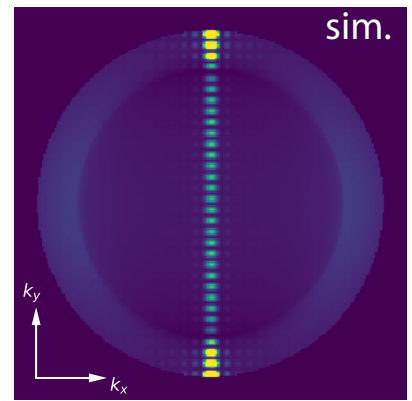


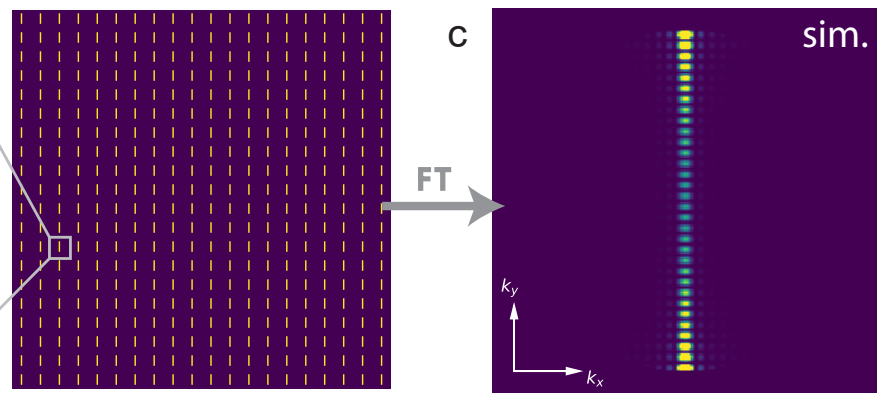

e

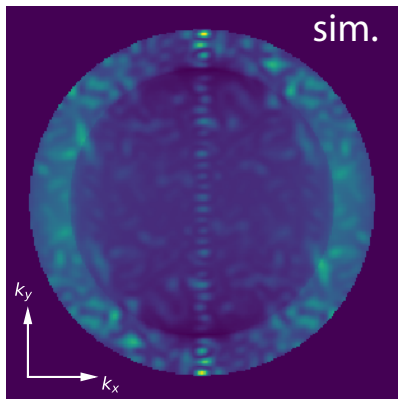

f

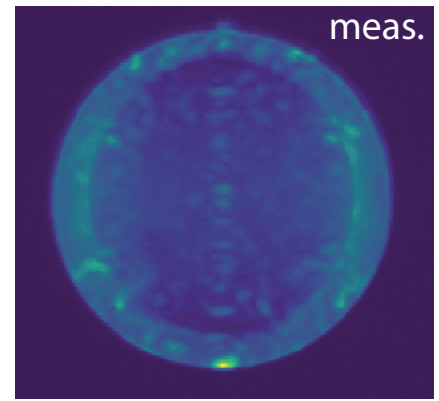

Fig. S 6. Noise study of the bare gold array. (a) Simulated electric field intensity distribution of the rod plasmonic resonance. (b) Illustration of the spatial arrangement of rods forming a 20 x 20 array. (c) Calculation of k-space of the 2D array assembly by a two-dimensional Fourier transformation. (d) Resulting k-space for a Gaussian excitation for dipoles on a glass substrate, supplemented by incoherent background (origin explained in the main text). (e) Final k-space calculation by employing random spatial fluctuations of the amplitude of individual rods (f) Measured SHG emission k-space of a bare gold array system.

Noise features in the background of the measurements (Figure 3b) motivated a numerical study of the SHG emission pattern of a bare gold array. The SHG emission measured at the back focal plane is shown in Figure S6f. For resonant excitation the emission is suppressed as described in the main text.

In order to assemble a model we look at the resonance intensity distribution for a single rod as shown in Figure S6a. The absolute value of the SHG electric field on the rod surface is assumed to have a similar distribution to the intensity of the excited resonant field due to the square relation between the two fields. In order to form complex fields for the calculation we assume a difference of phase $\pi$ between the two rod ends. Hence, an array of complex fields can be assembled as illustrated in Figure S6b. The k-space of the array SHG emission is then calculated with a two-dimensional Fourier transformation (FT). Figure S6c shows the absolute value of the field calculated in k-space. To further match the conditions of the measurement to the simulation we take into account the Gaussian spatial distribution of the excitation beam. Moreover, we multiply the resulting complex k-space with the corresponding far-field emission of a dipole on a glass substrate. Finally, we add incoherent background in a ratio with the coherent emission estimated by spectral measurements (Figure S2a). These modifications lead to the resulting k-space shown in Figure S6d. The ring in high $\left|k_{x, y}\right|$ values is formed due to the air-glass interface that favors emission in higher angles.

Finally, real-space measurements have shown that the emission is fluctuating between rods due to geometrical imperfections. By statistically evaluating this fluctuation we calculated an expected $20 \%$ deviation around a mean value. Embedding this random spatial fluctuation in the simulation in form of a normal statistical distribution we receive the k-space of the model accommodating the noise that we observe in the measurements as shown in Figure S6e. These results lead us to conclude that the residual emission of the bare gold array measured is a product of the aforementioned noise sources. 